\title{
Spontaneous coronary artery dissection with hypothyroidism in a 45-year-old
}

\section{man: A case report}

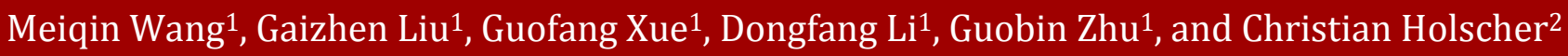

1. Neurology Department, The Second Affiliated Hospital of Shanxi Medical University, Taiyuan, Shanxi, PR China

2. School of Biomed Sciences, Ulster University, Coleraine, United Kingdom

\section{CASE STUDY}

Please cite this paper as: Wang M, Liu G, Xue G, Li D, Zhu G, Holscher C. Spontaneous coronary artery dissection with hypothyroidism in a 45-year-old man: A case report. AMJ 2018;11(12):561-564.

https://doi.org/10.21767/AMJ.2018.3528

\section{Corresponding Author:}

Dongfang Li

Department of Neurology, The Second Affiliated Hospital of Shanxi Medical University, Taiyuan, Shanxi, PR China

Email: lidongfang898@163.com

\section{ABSTRACT}

Spontaneous coronary artery dissection (SCAD) is a rare cause of acute coronary syndrome in young persons, rarely found in men. There are currently no known direct causes of this condition, although some correlations have been found. We report the case of a 45-year-old man with unstable angina pectoris secondary to spontaneous diffuse spiral dissection in the right coronary artery. In addition, the patient was diagnosed with other autoimmune related diseases, for example hypothyroidism, chronic atrophic gastritis, lung emphysema, which suggesting that autoimmune mechanism is an important mechanism for the occurrence of SCAD.

\section{Key Words}

Spontaneous coronary artery dissection, unstable angina pectoris, hypothyroidism, autoimmunity

\section{Implications for Practice:}

\section{What is known about this subject?}

SCAD is a rare cause of acute coronary syndrome that is commonly found in women. Generally severe symptoms and sudden cardiac death occurs in SCAD.

\section{What new information is offered in this case study?} Autoimmunity is a risk factor for SCAD, especially in male patient.

3. What are the implications for research, policy, or practice?

SCAD should always be considered when the patients have acute coronary syndrome and autoimmune related diseases, regardless of age.

\section{Background}

Spontaneous coronary artery disease (SCAD) is an infrequent condition of acute coronary syndrome. There are currently no known direct causes of this condition, although some correlations have been found. We report the case of a 45-year-old man with unstable angina pectoris caused by SCAD, suffered with several kinds of autoimmune diseases, which may suggests that autoimmune mechanism plays an important role in the occurrence of SCAD.

\section{Case details}

A 45-years-old man with episodes of chest pain for 2 months was admitted to our hospital for treatment on January 29, 2018. In 2008, sudden chest pain occurred after drinking, showing squeezing pain, accompanied by sweating and dyspnoea, renewing shoulder and back radiating pain, which spontaneously stopped after 30 minutes. The above symptoms did not recur. In the past 2 months, the patient had a recurrent chest pain, so he was first seen in the respiratory department. Lung function tests showed moderate reduction of diffusion function, and the chest $X$ ray showed an increased heart shadow. He was suggested to our institution for treatment. There is a remarkable history of risk factors of coronary atherosclerotic disease in this patient. He has a history of smoking for 30 years and drinking for more than 20 years. The physical examination 
shows that $\mathrm{P} 80 / \mathrm{min}, \mathrm{BP} 134 / 87 \mathrm{mmHg}$, and the $3 / 6$ systolic blow-like murmur in the apex region. Laboratory plasma analysis showed: homocysteine $27.3 \mathrm{umol} / \mathrm{L}$, serum levels of free thyroxine $17.06 \mathrm{pmol} / \mathrm{L}$, anti-thyroglobulin antibody $63.31 \mathrm{U} / \mathrm{mL}$, anti-thyroid peroxidase antibody $467.201 \mathrm{U} / \mathrm{mL}$ (Table 1), myocardial markers are normal. ECG showed: pathological Q waves of II, III, aVF lead, high T wave of V2V5 lead (Figure 1). Cardiac ultrasonography showed segmental abnormalities in the left ventricular wall, prolapse of the anterior segment of the mitral valve, incomplete closure (moderate), increase of left atrium and left ventricle.

It needs to be emphasized that he was diagnosed with hyperthyroidism in 2003, after I-131 radiotherapy diagnosed hypothyroidism in 2013. His mother and sister suffer from hypothyroidism. He also was diagnosed with lung emphysema, bullae in September 2016. He received treatment in a local hospital for abdominal distension and underwent gastroscopy, which revealed chronic atrophic gastritis associated with erosion and duodenal inflammation in June 2017. Chest CT showed: pulmonary emphysema, lung bullae, bronchiectasis and mild pulmonary interstitial fibrosis in the right lung bottom (Figure 2).

The patient was preliminary diagnosed with acute coronary syndrome, unstable angina, old myocardial infarction, hypothyroidism, chronic atrophic gastritis, pulmonary emphysema, and lung bullae. Dual anti-platelet therapy (clopidogrel and aspirin) was initiated. After a week of conservative treatment, no more chest pain occurred. On February 5, 2018, his coronary angiography showed that the right coronary artery was full of diffuse spiral intercalation (Figure 3). Therefore he was diagnosed with unstable angina caused by spontaneous coronary artery dissection. Indicated medical treatment, including aspirin, beta-blocker, ACE inhibitor, levothyroxine sodium was given. He was discharged and his chest pain did not reoccur for 6 months of follow-up.

\section{Discussion}

Spontaneous coronary artery dissection (SCAD), described as the dissection between the layers of media and adventitia in the coronary artery wall, has the histopathological characteristics of subadventitial dissection, hematoma formation in the cross-section of the coronary artery, and mixed inflammatory cell infiltration dominated by eosinophils in the adjacent adipose tissue. ${ }^{1}$ Among patients undergoing coronary angiography, it was estimated that 0.2 per cent-1.1 per cent had SCAD, ${ }^{2,3}$ and more commonly found in women rather than men, with a ratio of $2-3: 1 .^{4}$ In male patients, the dissection is more common in the right coronary artery. This is consistent with the patient manuscript reported.

The aetiology of SCAD is multifactorial. There is often a predisposing arteriopathy, with or without precipitating stress events. Arteriopathy causes include the peripartum state, connective tissue disorders, systemic inflammatory conditions, coronary artery spasm, or idiopathic events. ${ }^{1}$ Conditions such as systemic lupus erythematosus, rheumatic coronary arteritis, autothyroiditis, hepatitis C, gastritis, presumably may be related to chronic inflammation of the coronary artery. ${ }^{5}$ In addition, oral contraceptives, clonidine, caberin, homocysteineemia, $\alpha 1$ antitrypsin deficiency, protease overactivity, and myocardial bridging may cause SCAD., ${ }^{6,7}$ The lack of $\alpha 1$-antitrypsin can lead to dysfunction of multiple organs throughout the body. The main pulmonary manifestations are emphysema and bronchiectasis. It is also reported that $\alpha 1$-antitrypsin deficiency is related to the occurrence of vasculitis and aneurysms. ${ }^{8}$ It should be pointed out that our patient was diagnosed with emphysema and large bulla at the same time. Lung diffusion function decreased slightly. Alpha-1antitrypsin deficiency is the common etiologic basis of both diseases, so it is hypothesized that one of the causes of the right ductal dissection in this patient may be $\alpha 1$-antitrypsin deficiency. Our patient denied further examination.

The autoimmune disease pathology of arterial vasculature is the accumulation of auto-antibodies in various layers of the vessel wall, such as carotid artery dissection and aortic aneurysm, suggesting that the autoimmune mechanism is the pathogenesis of SCAD. Non-epithelial tissues such as thyroid and epithelial tissue proteins cross-react with arterial wall tissue, causing arterial wall damage. The patient in our case suffers from hypothyroidism and chronic atrophic gastritis, further suggesting that autoimmune mechanism may be an important mechanism for the occurrence of coronary artery dissection. Significant history of disease in our case was chest pain and the cardiac enlargement, so we inferred that the spontaneous dissection of the right coronary artery may result in the occurrence of myocardial infarction 10 years ago. Considering the slow progression of the dissection, the reason for this hospitalization may be that the recurrence of coronary artery dissection affects the angina pectoris.

At present, there is no relevant guideline for the treatment of SCAD. The treatment strategy mainly depends on the clinical symptoms of the patient, the size of the dissection, the size of the ischemic myocardium and other factors. The 
main treatment methods are drug treatment, percutaneous coronary intervention $(\mathrm{PCl})$ and coronary artery bypass grafting $(C A B G)$. For patients with stable coronary flow, a single antiplatelet, $\beta$-blockers, and statins can be prescribed. Statin therapy is only applicable to patients with dyslipidaemia. If patients have left ventricular dysfunction, $\beta$-blockers and angiotensin-converting enzyme inhibitors may be used. If the patient progresses with vascular occlusion, hemodynamic instability, significant symptoms, and ischemia, emergency $\mathrm{PCl}$ and $\mathrm{CABG}$ can be considered from the left main extension to the anterior descending and circumflex branches. ${ }^{9}$ The patient in this case report received aspirin, metoprolol, atorvastatin, candesartan sulphate tablets, levothyroxine sodium, and other medications, such as trimetazidine and folic acid tablets. Because of the right coronary artery was full of diffuse spiral intercalation, $\mathrm{PCl}$ and $\mathrm{CAGB}$ were impracticable, so we chose the medical approach. His chest pain did not reoccur after six months of follow-up.

\section{Conclusion}

Direct causes of SCAD are still unclear, our case illustrates that both $\alpha 1$-antitrypsin deficiency and autoimmune mechanism may play important part in the evolution of SCAD. That probably provides a direction for the mechanism and treatment research of SCAD.

\section{References}

1. Saw J. Spontaneous coronary artery dissection. Can J Cardiol. 2013;29:1027-33.

2. Mortensen $\mathrm{KH}$, Thuesen L, Kristensen IB, et al. Spontaneous coronary artery dissection: a Western Denmark Heart Registry study. Catheter Cardiovasc Interv. 2009;74:710-7.

3. Vanzetto G, Berger-Coz E, Barone-Rochette G, et al. Prevalence, therapeutic management and medium-term prognosis of spontaneous coronary artery dissection: results from a database of 11,605 patients. Eur J Cardiothorac Surg. 2009;35:250-4.

4. McCann AB, Whitbourn RJ. Spontaneous coronary artery dissection: a review of the etiology and available treatment options. Heart Vessels. 2009;24(6):463-5.

5. Amelia Yip, Jacqueline Saw. Spontaneous coronary artery dissection-A review. Cardiovasc Diagn Ther. 2015;5(1):37-48

6. Mehta NK, Malkani S, Ockene I. Spontaneous coronary artery dissection during cabergoline therapy. Tex Heart Inst J. 2012;39(1):92-94.

7. Melez IE, Arslan MN, Melez DO, et al. Spontaneous coronary artery dissection report of 3 cases and literature review hormonal, autoimmune, morphological factors. Am J Forensic Med Pathol. 2015;36(3):188-192.

8. Stoller JK, Aboussouan LS. Alpha-1-antitrypsin deficiency. Lancet. 2005;365:2225-2236.

9. Tweet MS, Gulati R, Hayes SN. What clinicians should know about spontaneous coronary artery dissection. Mayo Clin Proc. 2015;90(8):1125-1130.

\section{ACKNOWLEDGEMENTS}

The authors are indebted to all personnel in The Second Affiliated Hospital of Shanxi Medical University, especially in cardiology department, the catheterisation laboratory of the hospital.

\section{PEER REVIEW}

Not commissioned. Externally peer reviewed.

\section{CONFLICTS OF INTEREST}

The authors declare that they have no competing interests.

\section{FUNDING}

The University of Social Welfare and Rehabilitation Sciences

\section{PATIENT CONSENT}

The authors, Wang M, Liu G, Xue G, Li D, Zhu G, Holscher C, declare that:

1. They have obtained written, informed consent for the publication of the details relating to the patient(s) in this report.

2. All possible steps have been taken to safeguard the identity of the patient(s).

3. This submission is compliant with the requirements of local research ethics committees. 
Figure 1: pathological Q waves of II, III, aVF lead, high T wave of V2-V5 lead

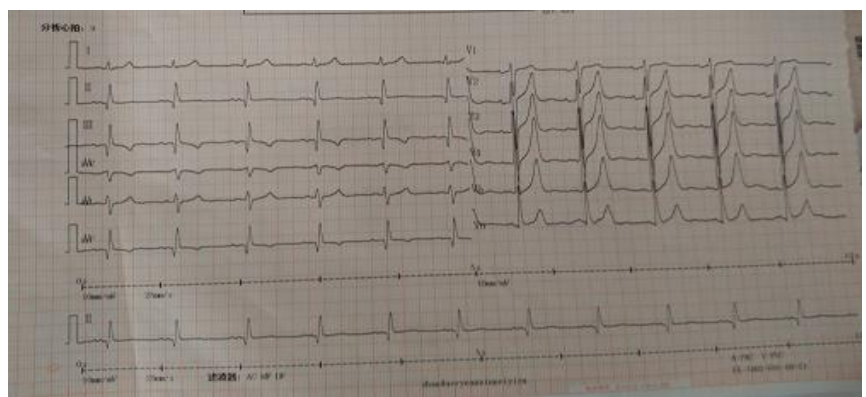

Table 1: Laboratory plasma analysis showed: serum levels of free thyroxine $17.06 \mathrm{pmol} / \mathrm{L}$, anti-thyroglobulin antibody $63.31 \mathrm{U} / \mathrm{mL}$, anti-thyroid peroxidase antibody $467.201 \mathrm{U} / \mathrm{mL}$

\begin{tabular}{|l|l|l|l|}
\hline \multicolumn{3}{|l|}{ Laboratory index Test result unit reference interval } \\
\hline $\begin{array}{l}\text { Serum free } \\
\text { triiodothyrosine (FT3) } \\
\text { Serum free thyroxine } \\
\text { (FT4) }\end{array}$ & 4.58 & Pmol/L & $3.8--6.0$ \\
$\begin{array}{l}\text { High sensitivity } \\
\text { serum thyrotropin } \\
\text { (TSH) }\end{array}$ & 1.69 & Pmol/L & $7.86--14.41$ \\
$\begin{array}{l}\text { Thyroglobulin (TG) } \\
\text { Antithyroglobulin } \\
\text { antibody(TG-Ab) } \\
\text { Parathyroid } \\
\text { Hormone(PTH) }\end{array}$ & $0.04 \downarrow$ & $\mathrm{ng} / \mathrm{mL}$ & $1.15--130.77$ \\
$\begin{array}{l}\text { Anti-thyroid } \\
\text { peroxidase antibody }\end{array}$ & $\uparrow 3.30 \uparrow$ & $\mathrm{IU} / \mathrm{mL}$ & $<4.0$ \\
\hline
\end{tabular}

Figure 2: Chest CT showed: pulmonary emphysema, lung bullae, bronchiectasis and mild pulmonary interstitial fibrosis in the right lung bottom

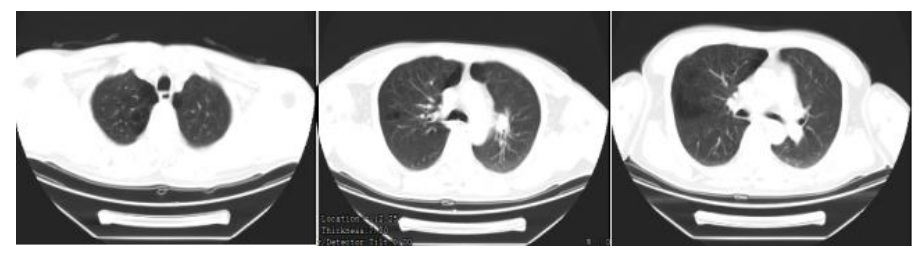

Figure 3: Angiography demonstrating spiral dissection of the right coronary artery

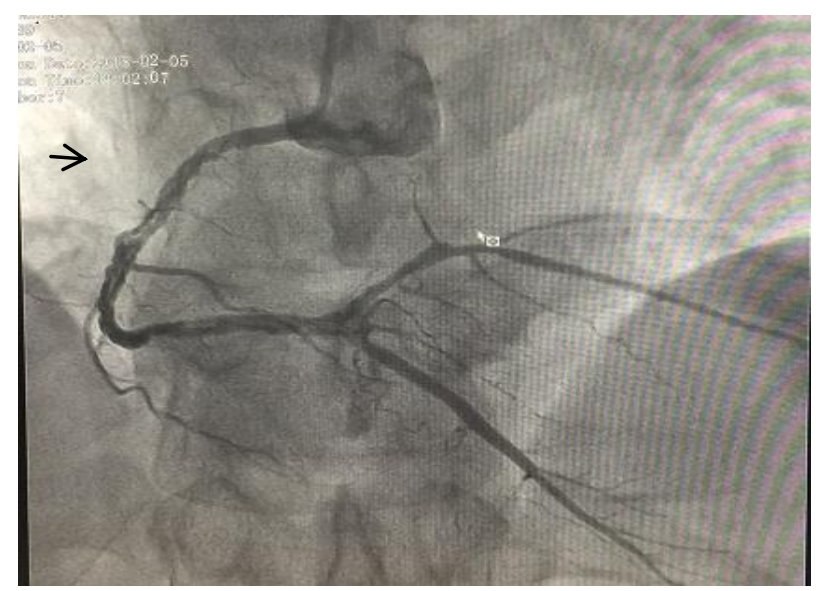

